\title{
Presynaptic Long-Term Potentiation in Corticothalamic Synapses
}

\author{
Manuel A. Castro-Alamancos and Maria E. Calcagnotto \\ Department of Neurology and Neurosurgery, Montreal Neurological Institute, McGill University, Montreal, Quebec \\ H3A2B4, Canada
}

\begin{abstract}
The thalamus and neocortex are two highly organized and complex brain structures that work in concert with each other. The largest synaptic input to the thalamus arrives from the neocortex via corticothalamic fibers. Using brain slices, we describe long-term potentiation (LTP) in corticothalamic fibers contacting the ventrobasal thalamus. Corticothalamic LTP is input-specific, NMDA receptor-independent, and reversible. The induction of corticothalamic LTP is entirely presynaptic and $\mathrm{Ca}^{2+}$-dependent. The expression of corticothalamic LTP is associated with a decrease in paired-pulse facilitation (PPF)
\end{abstract}

and blocked by an inhibitor of the cAMP-dependent protein kinase $A(P K A)$. Consistent with an involvement of cAMP and PKA, activation of adenylyl cyclase induced a synaptic enhancement that was associated with a decrease in PPF and occluded LTP. Corticothalamic LTP may serve to enhance the efficacy of cortico-cortical communication via the thalamus and/or to mediate experience-dependent long-term modifications of thalamocortical receptive fields.

Key words: thalamus; neocortex; synaptic plasticity; $c A M P$; protein kinase $A$; long-term potentiation; long-term depression
Almost all of the information that reaches the neocortex arrives from the thalamus via thalamocortical fibers. In return, the thalamus receives a massive feedback from the neocortex via corticothalamic fibers. The number of corticothalamic fibers is one order of magnitude larger than the number of thalamocortical axons (Sherman and Guillery, 1996), and cortical afferents are the most abundant input to the thalamus (Guillery, 1969). In the somatosensory system, the most corticothalamic fibers originate in layer VI and send reciprocal connections to the ventrobasal thalamus (ventroposteromedial and lateral nuclei), leaving a fiber collateral in the reticular nucleus (Zhang and Deschenes, 1997). Corticothalamic fibers from layer VI act on distal dendrites of thalamic neurons, forming a fine plexus with simple branching and fine synaptic boutons (Robson, 1983). In addition, there is a group of corticothalamic fibers that originates in layer $\mathrm{V}$ and reaches the posterior nucleus of the thalamus, without innervating the ventrobasal thalamus or reticular nucleus (Hoogland, 1991; Ojima, 1994; Bourassa et al., 1995; Levesque et al., 1996; Vidnyansky et al., 1996). Corticothalamic synapses use glutamate as their neurotransmitter, which activates both non-NMDA and NMDA ionotropic glutamate receptors (Deschenes and $\mathrm{Hu}$, 1990; Scharfman et al., 1990; Eaton and Salt, 1996; Kao and Coulter, 1997; Steriade et al., 1997; Turner and Salt, 1998). Metabotropic glutamate receptor-mediated responses have been described at these synapses in some studies (McCormick and von Krosigk, 1992; Eaton and Salt, 1996; Golshani et al., 1998), but not in others (Kao and Coulter, 1997; Turner and Salt, 1998). Repetitive stimulation of corticothalamic fibers produces shortterm facilitation of EPSPs in thalamic neurons (Frigyesi, 1972; Steriade and Wyzinski, 1972; Tsumoto et al., 1978; Deschenes

Received May 20, 1999; revised July 1, 1999; accepted Aug. 2, 1999.

This work was supported by the Medical Research Council of Canada, Fonds de la Recherche en Sante du Quebec, and the McGill University Research Development Fund. We thank Drs. Robert Malenka and John Robson for helpful comments on this manuscript.

Correspondence should be addressed to Dr. Manuel Castro-Alamancos, Montreal Neurological Institute, 3801 University Street, Room WB210, Montreal, Quebec H3A2B4, Canada.

Copyright (C) 1999 Society for Neuroscience 0270-6474/99/199090-08\$05.00/0 and $\mathrm{Hu}, 1990$; Lindstrom and Wrobel, 1990; Scharfman et al., 1990; McCormick and von Krosigk, 1992; Steriade and Timofeev, 1997).

The functional role of corticothalamic pathways is unknown. They may play a role in modifying the size, strength, and selectivity of thalamocortical receptive fields (Yuan et al., 1985; Diamond, 1995; Weinberger, 1995; Ergenzinger et al., 1998) and/or serve to establish cortico-cortical communication via the thalamus (Guillery, 1995). In all these instances, a mechanism for bidirectional activity-dependent long-term synaptic plasticity in corticothalamic synapses would be advantageous. It would provide the capacity to modify the effectiveness with which the neocortex can influence the thalamus. The most characteristic forms of long-term synaptic plasticity are long-term potentiation (LTP) and long-term depression (LTD). Both LTP and LTD have been studied in numerous synapses (Bliss and Collingridge, 1993; Bear and Malenka, 1994; Nicoll and Malenka, 1995), but they have yet to be described in the thalamus. We therefore performed a series of experiments to explore long-term synaptic plasticity in corticothalamic fibers.

\section{MATERIALS AND METHODS}

Thalamocortical slices were prepared from adult ( $\geq 7$ weeks) BALB/C mice according to the methods described by Agmon and Connors (1991). Slices were cut in ice-cold buffer using a vibratome and kept in a holding chamber for a least $1 \mathrm{hr}$. Experiments were performed in an interface chamber at $32^{\circ} \mathrm{C}$. The slices were perfused constantly $(1-1.5 \mathrm{ml} / \mathrm{min})$ with artificial CSF (ACSF) containing (in $\mathrm{mm}$ ): $\mathrm{NaCl} 126, \mathrm{KCl} 3$, $\mathrm{NaH}_{2} \mathrm{Po}_{4} 1.25, \mathrm{NaHCO}_{3} 26, \mathrm{MgSO}_{4} 7 \mathrm{H}_{2} \mathrm{O} 1.3$, dextrose 10 , and $\mathrm{CaCL}_{2}$ $2 \mathrm{H}_{2} \mathrm{O}$ 2.5. The ACSF was bubbled with $95 \% \mathrm{O}_{2}$ and $5 \% \mathrm{CO}_{2}$. Synaptic responses were induced using a concentric stimulating electrode (Frederick Haer Co.) placed in the thalamic radiation. The stimulus consisted of a $200 \mu \mathrm{sec}$ pulse of $<50 \mu \mathrm{A}$. The ventrobasal thalamus was easily and clearly identifiable with a dissecting microscope. Field recordings were made using a low-impedance pipette $(\sim 0.5 \mathrm{M} \Omega)$ filled with ACSF or with ACSF containing $400 \mu \mathrm{M}$ bicuculline methbromide (BMI). Intracellular recordings were performed using sharp electrodes (80-120 M $\Omega$ ) filled with $3 \mathrm{~m}$ potassium-acetate. The test stimulus was delivered at 0.05 $\mathrm{Hz}$ and was either single or a pair with a $50 \mathrm{msec}$ interstimulus interval to evaluate paired-pulse facilitation (PPF). The data are expressed as mean \pm SEM as a percentage of the baseline amplitude or slope. For 
single experiments, which represent typical examples, every response at $0.05 \mathrm{~Hz}$ is displayed.

The following drugs were stored as concentrated stock solutions and were diluted to the desired concentration using ACSF: $50 \mathrm{~mm}$ forskolin and 5 mM 1,9-dideoxyforskolin (dissolved in DMSO; Research Biochemicals, Natick, MA), $50 \mathrm{~mm}$ Rp-cAMPs (dissolved in water; Biomol, Plymouth Meeting, PA), $20 \mathrm{~mm}$ 6-cyano-7-nitroquinoxaline-2,3-dione disodium (CNQX) (dissolved in water; Research Biochemicals), $50 \mathrm{~mm}$ 土-2-amino-5-phosphonovaleric acid (APV) (dissolved in water; Research Biochemicals), 40 mm BMI (dissolved in water; Sigma, St. Louis, MO), $50 \mathrm{~mm}$ cyclothiazide (dissolved in DMSO; Research Biochemicals), and $\alpha$-methyl-4-carboxyphenylglycine (MCPG) (dissolved in the ACSF to $1 \mathrm{~mm}$; Research Biochemicals). All drugs were tested using bath application, although some drugs were also tested with local application. BMI was applied in either the bath $(40 \mu \mathrm{M})$ or the extracellular recording pipette by filling the recording pipette with ACSF containing BMI (400 $\mu \mathrm{M})$. CNQX and APV were applied in the bath $(10-20$ and $50-100 \mu \mathrm{M}$, respectively) or by using a low-impedance $(\sim 0.5 \mathrm{M} \Omega)$ pipette filled with ACSF containing these drugs (100 and $250 \mu \mathrm{M}$, respectively). The drug-containing pipette was placed adjacent $(\sim 500 \mu \mathrm{m})$ to the recording electrode, and the action of the drugs was monitored from the recording electrode.

\section{RESULTS}

\section{LTP in corticothalamic fibers}

Field and intracellular potentials were recorded from neurons of the ventrobasal thalamus in brain slices of adult mice (Fig. 1A). Orthodromic stimuli applied to the thalamic radiation evoked a negative potential in field recordings from populations of neurons and an EPSP in intracellular recordings from individual neurons of the ventrobasal thalamus (Fig. $1 B$ ). The field and intracellular EPSPs reflected a monosynaptic excitatory connection between corticothalamic fibers and neurons in the ventrobasal thalamus. This assertion is based on the following arguments. (1) The ventrobasal thalamus does not contain GABAergic interneurons, but it receives GABAergic input from the reticular nucleus (nRT), which is activated by corticothalamic fibers. To avoid the contribution of disynaptic IPSPs on corticothalamic responses, $\mathrm{GABA}_{\mathrm{A}}$ receptors were blocked locally by including BMI (400 $\mu \mathrm{M})$ in the low-impedance $(\sim 0.5 \mathrm{M} \Omega)$ extracellular recording electrode or in the bath $(40 \mu \mathrm{M})$. (2) Stimulation of the thalamic radiation may activate thalamocortical fibers and thus the neocortex. To ensure that activity in cortical circuits did not feed back to the thalamus, we severed all connections between thalamus and neocortex with a cut just below the cortical white matter (Fig. 1A). (3) Stimulation of the thalamic radiation could antidromically discharge neurons in the ventrobasal thalamus by directly activating their axons. However, antidromic activation cannot contribute to the recorded EPSPs because of the lack of recurrent connections between thalamic ventrobasal neurons. (4) Application of AMPA and NMDA receptor antagonists abolishes the field potential (see Fig. 5A). A short-latency (1-2 msec from the stimulus artifact) negativity resistant to glutamate receptor antagonists was observed in many extracellular recordings (see Fig. 5), and it was abolished by TTX ( $1 \mu \mathrm{M}$; data not shown). This short-latency non-synaptic component, the fiber volley, was monitored in many experiments as an index of fiber excitability. The field potential was also dependent on extracellular $\mathrm{Ca}^{+2}$ (see Fig. 5). (5) Consistent with a monosynaptic connection, both the intracellular and extracellular EPSPs have a constant and short latency of $\sim 3 \mathrm{msec}$, and both follow high-frequency $(50 \mathrm{~Hz})$ stimulation without failure. As for other monosynaptic excitatory connections, pairedpulse stimulation produces strong facilitation in both the field and intracellularly recorded EPSPs (Fig. $1 B$ ). Facilitation is a consistent finding among studies that test corticothalamic pathways (Frigyesi, 1972; Steriade and Wyzinski, 1972; Tsumoto et al.,
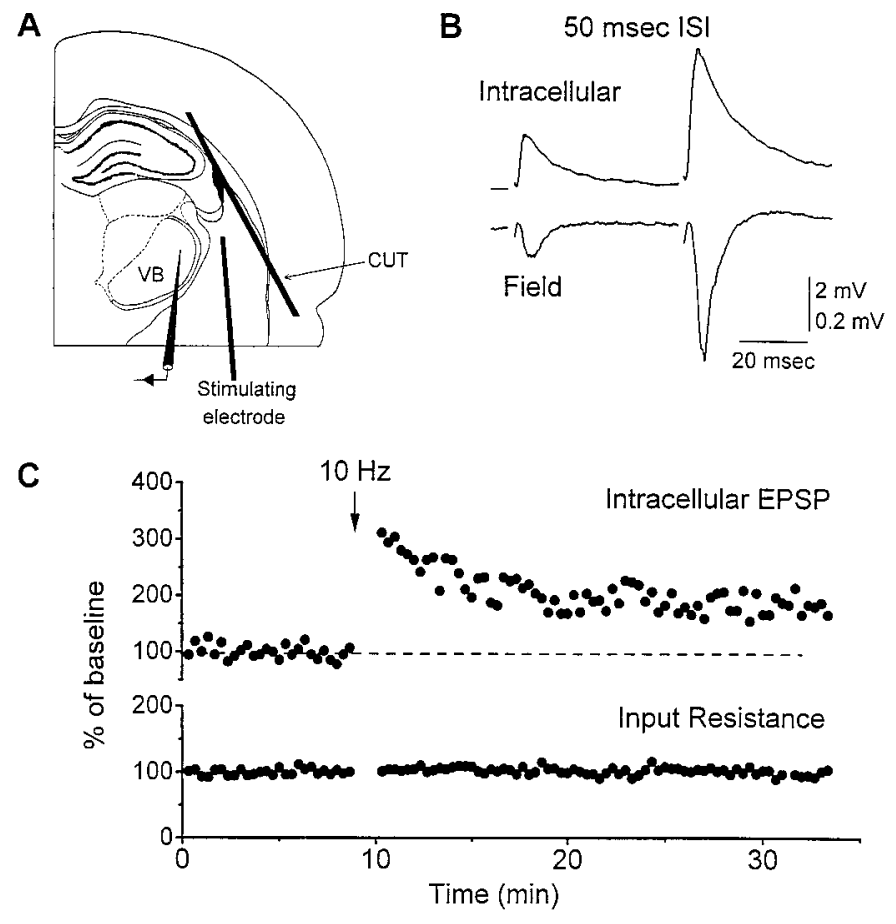

Figure 1. Intracellular and field corticothalamic EPSPs and LTP. $A$, Diagram of the corticothalamic slice preparation. The typical positions of the stimulating and recording electrodes are shown. The stimulating electrode is placed in the thalamic radiation. The recording electrode is placed in the ventrobasal thalamus $(V B)$. To ensure that activity in neocortical circuits did not feed back to the thalamus, all connections between thalamus and neocortex were severed with a cut below the cortical white matter. $B$, Paired-pulse facilitation of corticothalamic EPSPs. Intracellular and field EPSPs evoked in the ventrobasal thalamus in response to a pair of stimuli delivered in the thalamic radiation with a $50 \mathrm{msec}$ interstimulus interval (ISI). C, LTP of corticothalamic EPSPs. Effect of $10 \mathrm{~Hz}$ stimulation (600 pulses in 6 trains of 100 pulses each delivered with a $10 \mathrm{sec}$ interval between trains) on the amplitude of an intracellular EPSP recorded from the ventrobasal thalamus in response to stimulation of the thalamic radiation. The input resistance of the same neuron was also monitored with a $50 \mathrm{msec}$ current pulse. Test stimuli were applied at $0.05 \mathrm{~Hz}$, and all responses are displayed.

1978; Deschenes and Hu, 1990; Lindstrom and Wrobel, 1990; Scharfman et al., 1990; McCormick and von Krosigk, 1992; Steriade and Timofeev, 1997). The only corticothalamic input to the ventrobasal thalamus arises from neocortical neurons in layer VI (Bourassa et al., 1995). Based on these arguments, we conclude that the synaptic responses we recorded in response to stimulation of the thalamic radiation result from the depolarization of ventrobasal neurons caused by the release of glutamate from corticothalamic fibers originating in layer VI neurons.

We first explored the competence of corticothalamic EPSPs to undergo LTP. To induce LTP, 600 pulses were delivered in six trains of 100 pulses each with an interval between trains of $10 \mathrm{sec}$. Trains consisted of 1 or $10 \mathrm{~Hz}$. After $10 \mathrm{~Hz}$ stimulation, a lasting synaptic enhancement (LTP, >35 min) developed in both the field and the intracellular EPSP (Fig. 1C). Typically, $10 \mathrm{~Hz}$ stimulation produces a strong several-fold increase in the synaptic response, which decays within the next few minutes to a stable enhancement of $\sim 180 \%$ of the baseline. In a few experiments $(n=3)$, LTP was followed for $2 \mathrm{hr}$ without decrement. LTP is demonstrated in both the field and intracellular EPSPs (Figs. $1 C$, $2 A$ ). Intracellular recordings from ventrobasal thalamic neurons demonstrate that LTP is not associated with a change in input 
A
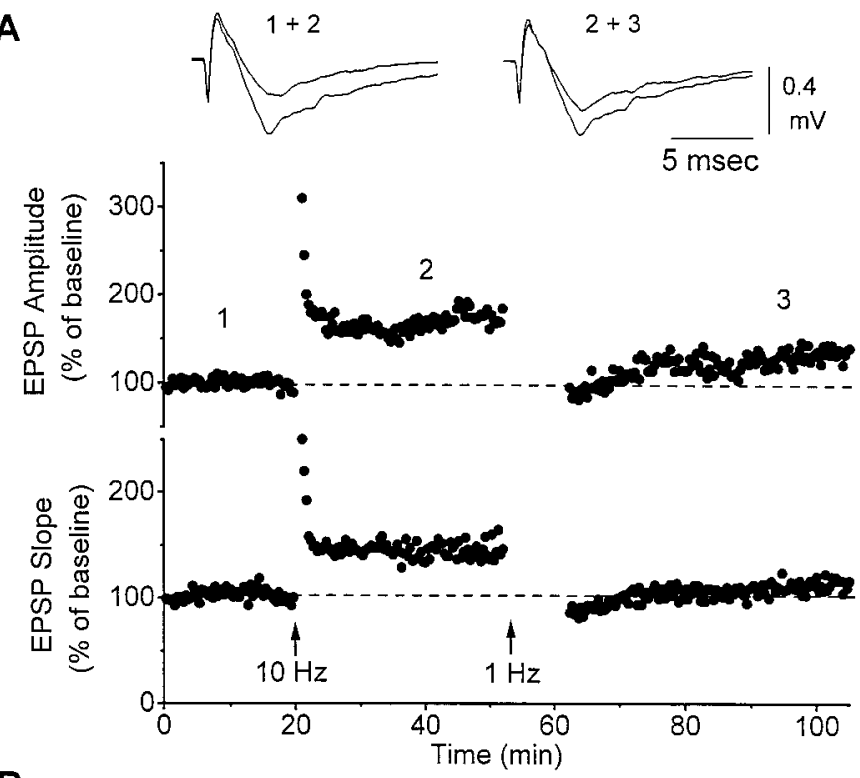

B

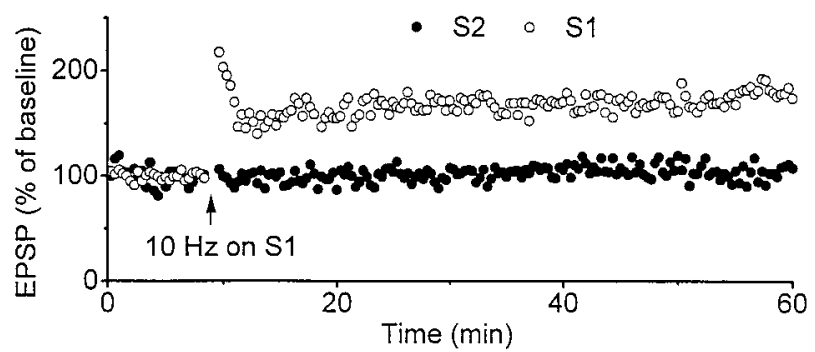

Figure 2. Corticothalamic LTP is reversible and input-specific. $A$, Stimulation $(10 \mathrm{~Hz} ; 600$ pulses) produces LTP that is expressed as a change in the slope and amplitude of the field potential. Application of $1 \mathrm{~Hz}$ stimulation (600 pulses) $30 \mathrm{~min}$ after LTP induction reverses LTP. The numbers in the graph correspond to the field recordings shown above. $B$, Responses were evoked by two stimulating electrodes placed in the thalamic radiation and recorded from a single electrode in the ventrobasal thalamus. Stimulation $(10 \mathrm{~Hz})$ delivered to one of the electrodes $(\mathrm{S} 1$; open circles) produced LTP but had no effect on the responses evoked from the other stimulating electrode ( 2 ; closed circles). The amplitude of the field potential was measured.

resistance (measured by applying a current pulse; $50 \mathrm{msec}, 0.2$ $\mathrm{nA} ; n=4)$ (Fig. $1 C$ ). During intracellular recordings $(n=10)$, the amplitude of the evoked EPSP was set at an amplitude that did not reach threshold to trigger a low-threshold calcium spike, and none of the neurons included in the study were antidromically activated with the intensities used. Field recordings established that LTP is not associated with a change in fiber excitability, as determined by measuring the fiber volley $(100 \pm 3 \%$ of the baseline fiber volley amplitude measured $35 \mathrm{~min}$ after LTP induction; $n=4$ ). After LTP has been induced, with $10 \mathrm{~Hz}$ stimulation, subsequent activation of the same corticothalamic fibers at $1 \mathrm{~Hz}$ (using the same number of stimuli) produces a lasting synaptic depression (LTD) or depotentiation that reverses LTP (Fig. 2A). For the remainder of the study, we investigated the properties of corticothalamic LTP induced by $10 \mathrm{~Hz}$ stimulation.

The next experiment sought to investigate whether LTP was input-specific. Two stimulating electrodes were placed in the thalamic radiation on two independent sets of fibers. A population of thalamic neurons was monitored using a single recording electrode. To ensure that the electrodes activated independent sets of fibers, we tested for paired-pulse interactions between them. When two stimuli are delivered with a $50 \mathrm{msec}$ interval through the same electrode, they activate the same set of fibers and produce robust synaptic facilitation. In contrast, if the first stimulus is delivered through one electrode and the second stimulus (50 msec latter) is delivered through another electrode, facilitation to the second stimulus would only occur if both electrodes activate the same set of fibers. Thus, by ensuring that facilitation to the second stimulus delivered through the second electrode does not occur, we can assume that the two electrodes activate different fibers. After determining that the two electrodes activated independent sets of fibers, we applied $10 \mathrm{~Hz}$ stimulation to one of them. LTP developed only in the pathway in which the $10 \mathrm{~Hz}$ stimulation was applied $(n=5)$ (Fig. $2 B)$. This indicates that corticothalamic LTP is input-specific.

Placing BMI locally in the recording pipette $(400 \mu \mathrm{M})$ or in the bath $(40 \mu \mathrm{M})$ tested the effect of blocking $\mathrm{GABA}_{\mathrm{A}}$ receptors on LTP induction. In other areas, such as the hippocampus (Schaffer collaterals) and the neocortex, the induction of LTP is eased by $\mathrm{GABA}_{\mathrm{A}}$ receptor block (Wigstrom and Gustafsson, 1983; CastroAlamancos et al., 1995). However, we found no significant effect of BMI on the induction of LTP in corticothalamic fibers (Fig. $3 B)$. The average enhancement in the presence of BMI was $183 \%$ of the baseline, which is not significantly different from the average synaptic enhancement observed without BMI $(n=5 ; 35$ min after LTP induction; $t$ test; NS) (Fig. $3 A$ ). Despite this finding, we used local BMI application for the remainder of the experiments to be certain that our manipulations were not mediated through changes in disynaptic $\mathrm{GABA}_{\mathrm{A}}$ IPSPs.

We then examined whether the induction of LTP required the activation of NMDA receptors or metabotropic glutamate receptors. LTP induction was not significantly affected by bath application $(100 \mu \mathrm{M})$ of the NMDA antagonist APV (Fig. 3C). Neither the initial large potentiation nor the persistent enhancement of the corticothalamic field potential were significantly affected in slices treated with APV compared with control slices $(n=5 ; 35$ min after LTP induction; $t$ test; NS). The effectiveness of APV was verified by demonstrating that Schaffer collateral LTP was blocked in the same slices $(100 \mathrm{~Hz}$ tetanus for $1 \mathrm{sec}$ repeated four times at $10 \mathrm{sec}$ intervals). LTP induction was also not significantly affected by bath application $(1 \mathrm{~mm})$ of the metabotropic glutamate receptor antagonist MCPG $(n=4 ; 178 \pm 9 \%$ of baseline responses; 35 min after LTP induction). Moreover, application of MCPG had no significant effect on the baseline field potential response (data not shown).

LTP was further characterized by testing its effects on PPF. PPF is a well defined presynaptic process in which residual $\mathrm{Ca}^{2+}$ influx after the first of two stimuli results in an enhancement of transmitter release in response to the second stimulus (Zucker, 1989). In the mossy fibers of hippocampus and in the parallel fibers of cerebellum in which LTP is presynaptic and not dependent on NMDA receptor activation, LTP leads to a persistent depression of PPF (Zalutsky and Nicoll, 1990; Salin et al., 1996). We found that LTP in corticothalamic fibers is accompanied by a persistent reduction of PPF (Fig. 4). The reduction in PPF was very large during the initial minute of strong potentiation after the $10 \mathrm{~Hz}$ stimulation. During the stable synaptic enhancement, PPF was also significantly reduced. The average PPF 35 min after LTP induction was $70 \pm 5 \%$ of the baseline $(n=5)$. Because PPF changes can be caused by changes in the desensitization of AMPA receptors (Wang and Kelly, 1996), we examined LTP and 
A

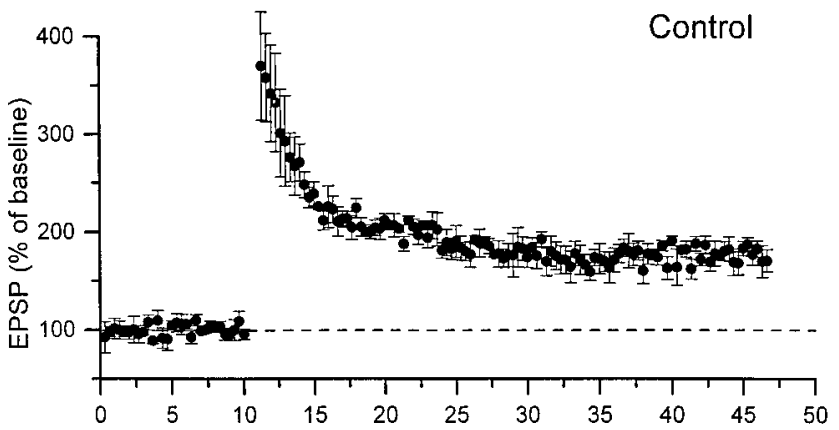

B

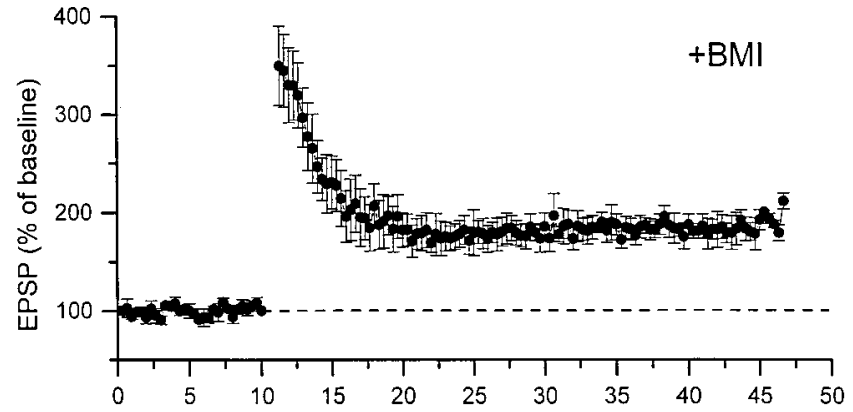

C

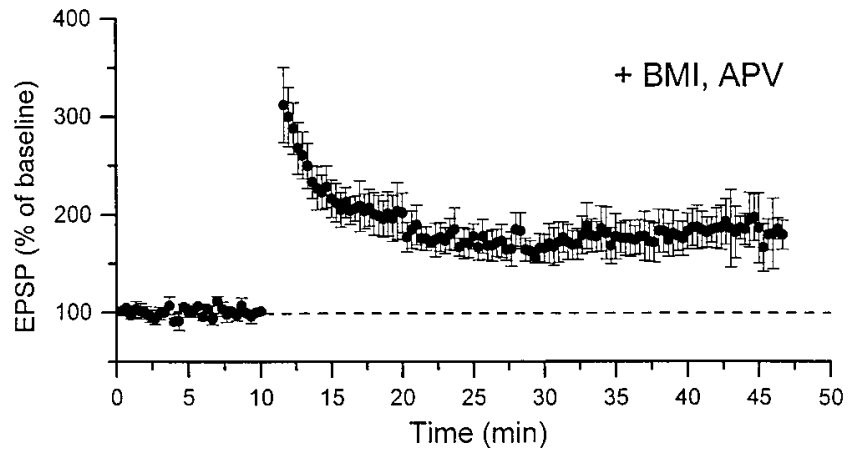

Figure 3. Corticothalamic LTP is not affected by NMDA receptor block or disinhibition. $A$, Summary of experiments $(n=5)$ showing the effects of $10 \mathrm{~Hz}$ stimulation on the amplitude of corticothalamic field potentials in normal ACSF (Control). B, Summary of experiments $(n=5)$ in which $10 \mathrm{~Hz}$ stimulation was applied in the presence of BMI $(400 \mu \mathrm{M})$ in the field recording pipette. $C$, Summary of experiments $(n=5)$ in which 10 $\mathrm{Hz}$ stimulation was applied in the presence of APV $(100 \mu \mathrm{M})$ in the bath. Data correspond to the amplitude of the field potential expressed as the mean \pm SEM of every response at $0.05 \mathrm{~Hz}$. Stimulation $(10 \mathrm{~Hz})$ was applied after a $10 \mathrm{~min}$ baseline.

PPF in the presence of cyclothiazide $(50 \mu \mathrm{M})$, which prevents the desensitization of AMPA receptors. In every case tested $(n=3)$, we observed that PPF was reduced during LTP in the presence of cyclothiazide (72 $\pm 7 \%$ of baseline) to a similar degree than control slices ( $70 \pm 5 \%$ of baseline). These results suggest that the expression of LTP in corticothalamic synapses is presynaptic.

\section{Corticothalamic LTP induction does not require postsynaptic activation but requires calcium}

The results show that corticothalamic LTP is input-specific, not dependent on the activation of NMDA receptors or disinhibition, and proceeds with a persistent occlusion of PPF. We next sought to test whether there is an actual postsynaptic involvement in the induction of corticothalamic LTP. To address this issue, we applied $10 \mathrm{~Hz}$ stimulation in the presence of NMDA and nonNMDA receptor antagonists $(100 \mu \mathrm{M}$ CNQX and $250 \mu \mathrm{M}$ APV were applied using a pipette placed adjacent to the recording
A

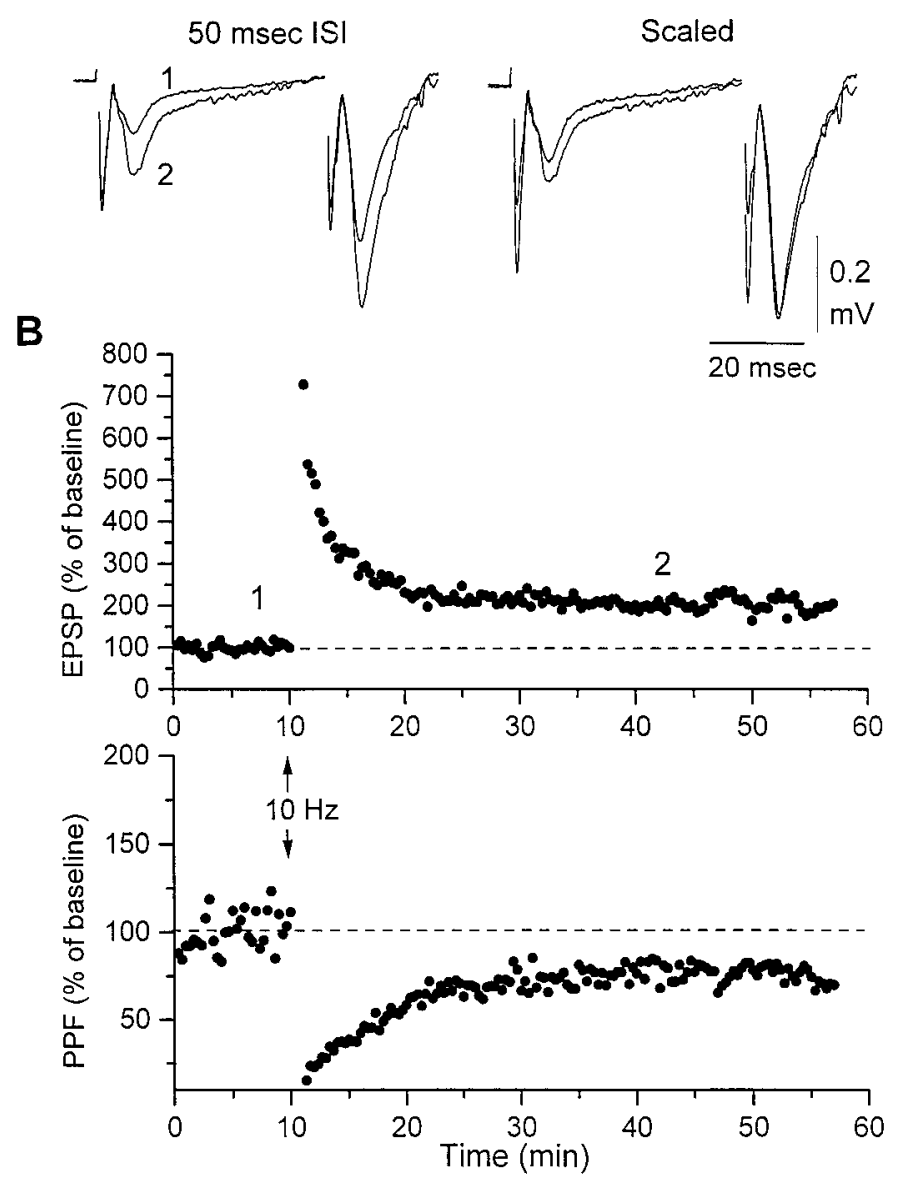

Figure 4. Corticothalamic LTP occludes PPF. A, Changes in pairedpulse facilitation during LTP. The field recordings show the effect of paired-pulse stimulation (50 msec interstimulus interval) before and 35 min after LTP induction. The field recordings correspond to the numbers shown in $B$. On the right, the second responses of the pair are scaled to reveal changes in PPF. Scaling is accomplished by changing the $y$-axis scale until the second responses of the pairs are equal in amplitude before and during LTP. B, Paired pulses $(50 \mathrm{msec})$ were delivered at $0.05 \mathrm{~Hz}$ before and after the induction of LTP. Shown is the amplitude of the field potential to the first pulse (top) and the change in PPF associated with LTP displayed as a percentage of the baseline PPF (bottom).

electrode). Figure 5 shows such an experiment. An initial application of the glutamate receptor antagonists completely blocks evoked postsynaptic activity but leaves intact the fiber volley. Upon removal of the antagonists, the responses recover to their baseline amplitudes. The antagonists were applied again, but in this case, $10 \mathrm{~Hz}$ stimulation was delivered during the receptor blockade. Upon removal of the antagonists, the synaptic response did not return to control levels but increased to a stable enhancement $(198 \pm 9 \%$ of baseline after $1 \mathrm{hr} ; n=5)$ (Fig. $5 B)$. Moreover, LTP induced during postsynaptic block was accompanied by a significant reduction of PPF. A subsequent block of postsynaptic activity, which revealed again the fiber volley, demonstrates that LTP is not accompanied by a change in fiber excitability (Fig. $5 A, C$ ). These results show that postsynaptic activity is not required for the induction of corticothalamic LTP.

The dependence of corticothalamic LTP on extracellular $\mathrm{Ca}^{2+}$ was also examined. Removal of $\mathrm{Ca}^{2+}$ from the $\operatorname{ACSF}\left(0 \mathrm{Ca}^{2+}\right.$ 
A

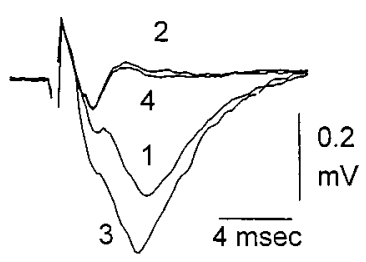

C

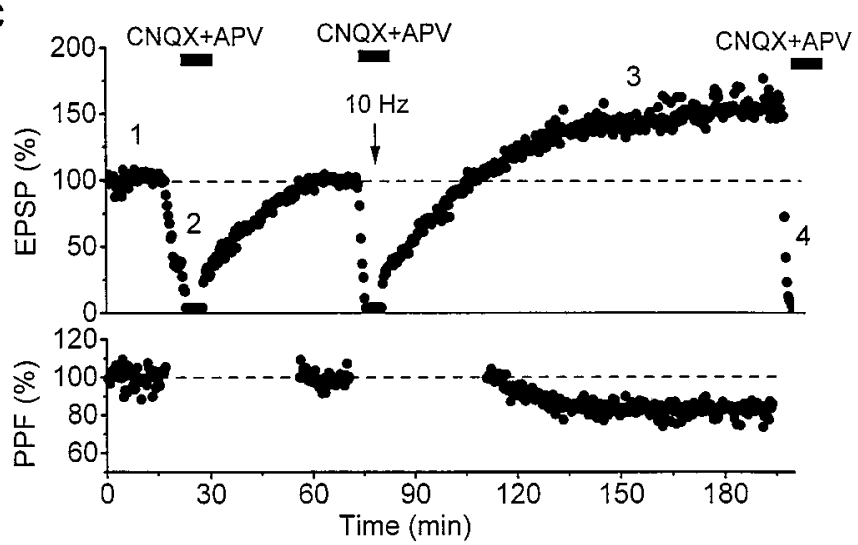

D

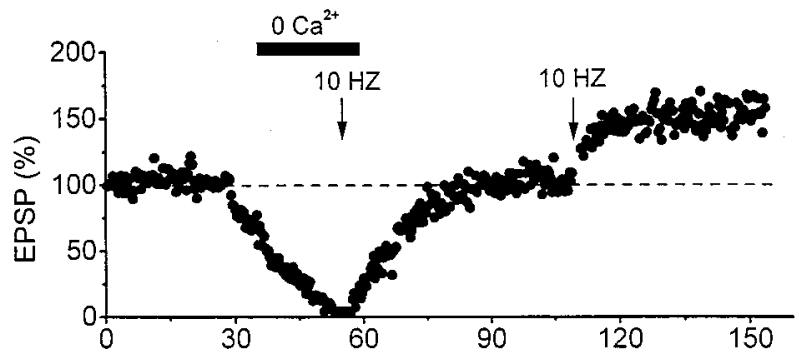

Figure 5. Corticothalamic LTP induction does not require postsynaptic activation but requires calcium. $A$, Field responses correspond to the experiment shown in C. B, Comparison of the effects of previous application of glutamate receptor antagonists (CNQX and APV) or $0 \mathrm{Ca}^{2+}$ ACSF during which $10 \mathrm{~Hz}$ stimulation was applied (black) or was not applied (white). Shown are the amplitudes of the responses (mean \pm SEM; $n=5-7)$ recorded $1 \mathrm{hr}$ after recovery from complete field EPSP block (induced by CNQX plus APV or by $0 \mathrm{Ca}^{2+} \mathrm{ACSF}$ ), represented as the percentage of the baseline amplitude (before field EPSP block). $C, \mathrm{~A}$ typical experiment showing that, after a brief application of CNQX and APV, which completely blocks postsynaptic responses (compare 1 and 2 in $A$ ), field EPSPs return to their baseline amplitudes. A subsequent application of CNQX and APV during which $10 \mathrm{~Hz}$ stimulation was applied produces LTP that is associated with a decrease in PPF. Subsequent application of CNQX and APV serves to demonstrate that the fiber volley did not change during LTP (compare 2 and 4 in $A$ ). $D$, Application of $10 \mathrm{~Hz}$ stimulation during complete block of synaptic transmission caused by $0 \mathrm{Ca}^{2+}$ ACSF did not produce LTP. A subsequent application of $10 \mathrm{~Hz}$ stimulation in the presence of extracellular $\mathrm{Ca}^{+2}$ induces LTP. Data are presented as the percentage of the baseline field potential amplitude.

and $4 \mathrm{mM} \mathrm{Mg}^{2+}$ ACSF) blocked corticothalamic synaptic transmission (Fig. 5D). Application of $10 \mathrm{~Hz}$ stimulation during complete block of synaptic transmission did not produce LTP; responses returned to baseline levels after restoration of $\mathrm{Ca}^{2+}$ (102 $\pm 6 \%$ of baseline; $n=7$ ) (Fig. $5 B)$. Subsequent application of $10 \mathrm{~Hz}$ stimulation in the presence of extracellular $\mathrm{Ca}^{2+}$ induces LTP (Fig. 5D). This finding, together with the lack of effect of blocking postsynaptic depolarization, indicates that a presynaptic rise in $\mathrm{Ca}^{2+}$ is necessary for the induction of LTP.
A

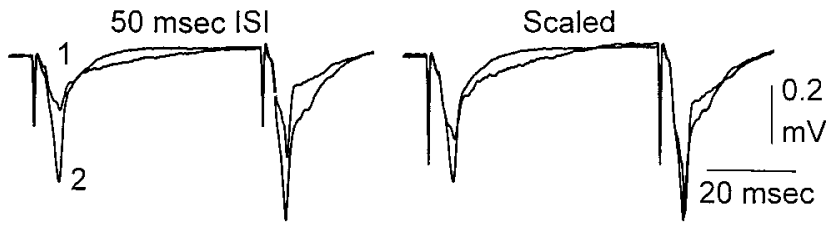

B

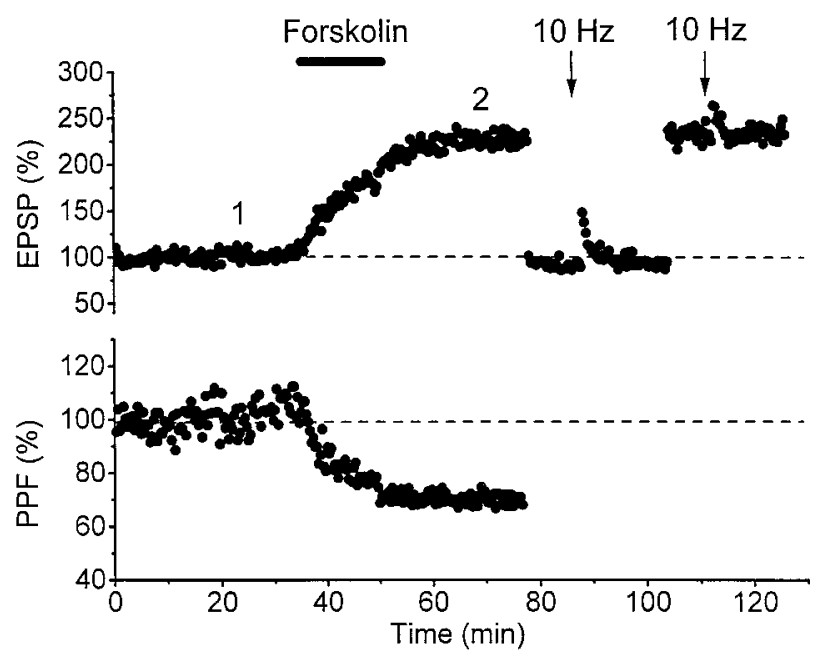

C

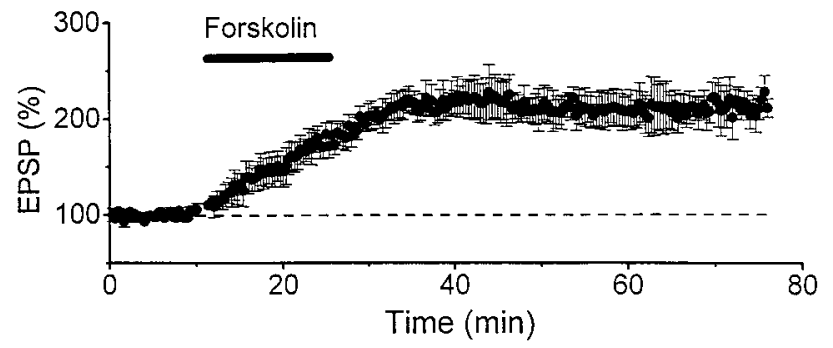

Figure 6. Forskolin enhances corticothalamic field potentials and occludes PPF and LTP. $A$, Change in paired-pulse facilitation during forskolin-induced enhancement. The field recordings represent the effect of two stimuli delivered with a $50 \mathrm{msec}$ interval (ISI) before and $35 \mathrm{~min}$ after forskolin-induced enhancement. The field recordings correspond to the numbers shown in $B$. On the right, the second responses of the pair are scaled to reveal changes in PPF. $B$, Paired-pulse stimulation $(50 \mathrm{msec})$ was delivered at $0.05 \mathrm{~Hz}$ before, during, and after application of forskolin (50 $\mu \mathrm{M})$. Plotted is the amplitude of the field potential to the first response (top) and the change in PPF (bottom), displayed as a percentage of the baseline PPF. During forskolin-induced enhancement, the evoked response was reduced to the baseline amplitude by decreasing the stimulus, and $10 \mathrm{~Hz}$ stimulation was applied. The stimulus amplitude was restored, and $10 \mathrm{~Hz}$ stimulation was delivered again. $C$, Summary of five experiments $($ mean \pm SEM) in which forskolin enhanced corticothalamic field potentials.

\section{Expression of corticothalamic LTP involves CAMP and protein kinase $\mathbf{A}$}

LTP, which is NMDA receptor-independent and induced by a presynaptic increase in $\mathrm{Ca}^{2+}$, has been described in hippocampal mossy fibers and cerebellar parallel fibers (Zalutsy and Nicoll, 1990; Huang et al., 1994; Weisskopf et al., 1994; Salin et al., 1996). These forms of LTP and a similar type found in the lateral amygdala (Huang and Kandel, 1998) have been shown to depend on CAMP. To test the role of cAMP in corticothalamic LTP, we applied forskolin, which directly activates adenylyl cyclase and elevates cAMP levels. Forskolin enhanced corticothalamic synaptic responses, and this enhancement was long-lasting (at least 2 hr) despite the brief application (15 min; $n=5$ ) (Fig. 6). Similar 
to LTP induced with $10 \mathrm{~Hz}$ stimulation, forskolin-induced enhancement was associated with a decrease in PPF and with no change in the fiber volley (Fig. 6). The forskolin analog dideoxyforskolin $(50 \mu \mathrm{M})$, which does not activate adenylyl cyclase but mimics other effects of forskolin, did not induce a lasting synaptic enhancement $(103 \pm 5 \%$ of baseline; $35 \mathrm{~min}$ after application; $n=3)$. Thus, direct activation of adenylyl cyclase mimics the effect of $10 \mathrm{~Hz}$ stimulation on corticothalamic fibers.

If forskolin-induced LTP and $10 \mathrm{~Hz}$-induced LTP share the same underlying mechanisms, elevation of cAMP by forskolin should occlude further potentiation by $10 \mathrm{~Hz}$ stimulation of corticothalamic fibers. To test this, we tried to induce LTP in corticothalamic fibers after forskolin had enhanced synaptic transmission. As shown in Figure 6, after application of forskolin, $10 \mathrm{~Hz}$ stimulation failed to induce LTP (106 $\pm 9 \%$ of baseline; 35 min after $10 \mathrm{~Hz}$ stimulation; $n=4)$. LTP was not observed either if the $10 \mathrm{~Hz}$ stimulation was applied after returning the field potential to the pre-forskolin baseline amplitude or if it was applied at the post-forskolin amplitude (Fig. 6). We were sure that these slices were capable of generating LTP because $10 \mathrm{~Hz}$ stimulation was tested before forskolin in a different set of corticothalamic fibers from the same slice or in other slices from the same animal, confirming that corticothalamic LTP could be generated.

Because cAMP elevation is able to induce LTP in corticothalamic fibers, we tested whether an inhibitor of protein kinase A (PKA) blocked LTP. Bath application of Rp-cAMPs $(50 \mu \mathrm{M})$, a potent and competitive PKA inhibitor, had no effect on baseline synaptic transmission; however, LTP was not generated by $10 \mathrm{~Hz}$ stimulation $(n=6)$ (Fig. 7). This effect was partially reversible in some experiments because application of $10 \mathrm{~Hz}$ stimulation after washout of the drug was able to induce LTP (130 $\pm 9 \%$ of baseline compared with $180 \%$ in control slices).

\section{DISCUSSION}

The present experiments reveal that corticothalamic fibers generate input-specific and reversible LTP in the ventrobasal thalamus. The results indicate that corticothalamic LTP is entirely presynaptic and appears to rely on the same mechanisms as LTP observed at hippocampal mossy fibers or cerebellar parallel fibers. The inability of glutamate receptor antagonists to impede the induction of corticothalamic LTP strongly suggests that this form of LTP is independent of postsynaptic depolarization or postsynaptic $\mathrm{Ca}^{2+}$ influx. However, extracellular $\mathrm{Ca}^{2+}$ is necessary for corticothalamic LTP, suggesting that a rise in presynaptic $\mathrm{Ca}^{2+}$ is required. The results also show that LTP in corticothalamic fibers is not dependent on NMDA receptor activation or disinhibition. This reinforces the notion that postsynaptic mechanisms are not involved in the induction of corticothalamic LTP. In pathways in which postsynaptic depolarization and postsynaptic $\mathrm{Ca}^{2+}$ entry are important (e.g., neocortex, Schaffer collaterals), LTP induction is strongly facilitated by disinhibition and blocked by NMDA receptor antagonists. The decrease in PPF during corticothalamic LTP provides further evidence that not only the induction but also the expression of this form of LTP is presynaptic and therefore caused by an increase in neurotransmitter release. The results also show that activation of adenylyl cyclase by forskolin causes a long-lasting potentiation, which decreases PPF and occludes LTP induced by $10 \mathrm{~Hz}$ stimulation. Moreover, application of a PKA inhibitor blocks the generation of corticothalamic LTP. Together, the results can be interpreted by a model put forward to explain LTP in hippocampal mossy fibers (Huang et al., 1994; Weisskopf et al., 1994). In this model, presynaptic $\mathrm{Ca}^{2+}$ entry
A

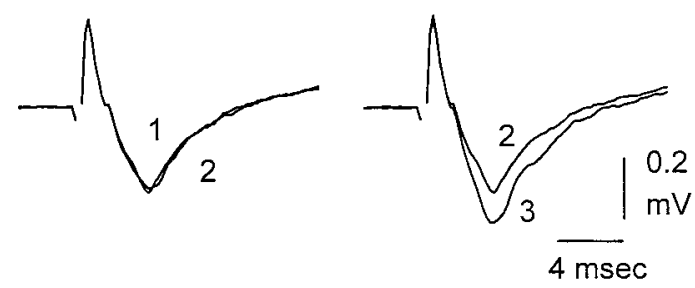

B
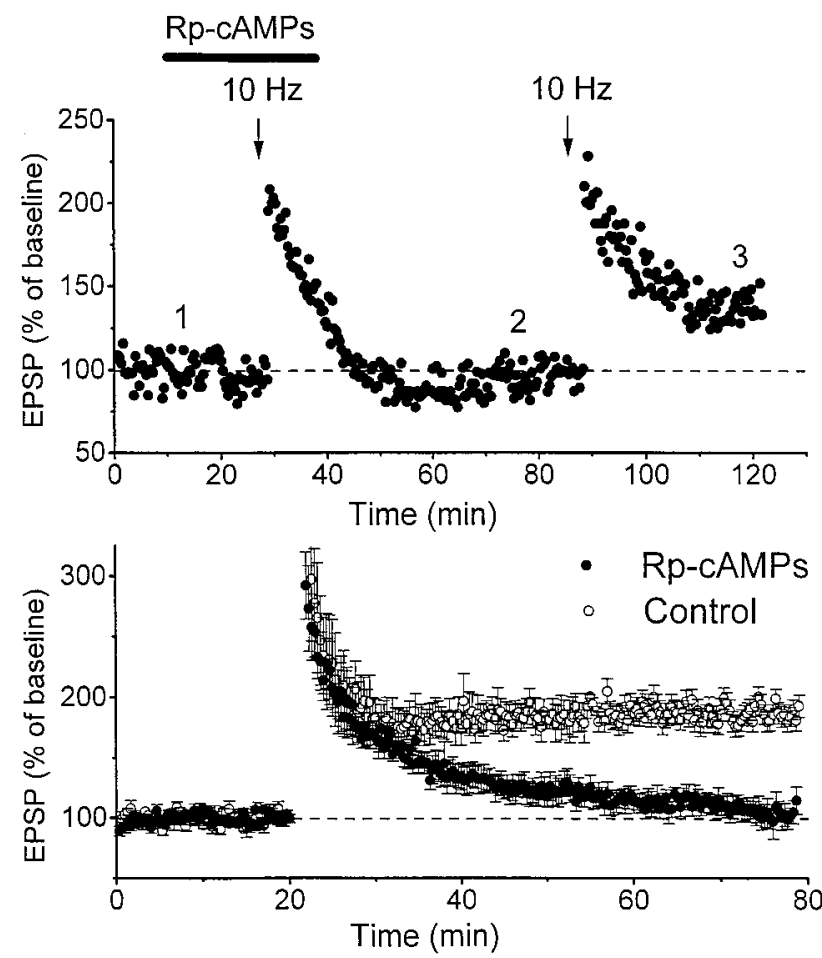

Figure 7. Corticothalamic LTP is blocked by a PKA inhibitor. $A$, The field recordings correspond to the experiment shown in $B . B, \mathrm{Rp}$-cAMPs, an inhibitor of PKA, blocked corticothalamic LTP. Responses were evoked at $0.05 \mathrm{~Hz}$, and Rp-cAMPs was included $(50 \mu \mathrm{M})$ in the bath without any effect on the baseline after a $25 \mathrm{~min}$ application of Rp-cAMPs $10 \mathrm{~Hz}$ stimulation was applied, and LTP was not induced. In this experiment, a subsequent application of $10 \mathrm{~Hz}$ stimulation $\sim 40$ min after Rp-cAMPs washout produced LTP. $C$, Summary of experiments in which $10 \mathrm{~Hz}$ stimulation was applied in control slices $(n=5)$ or in slices bathed in Rp-cAMPs $(n=6)$.

activates an adenylyl cyclase that, via cAMP and PKA, generates LTP through an increase in neurotransmitter release probability.

Presynaptic PKA-dependent mechanisms involved in the generation of LTP have been described in at least three different pathways: hippocampal mossy fibers (Huang et al., 1994; Weisskopf et al., 1994), parallel fibers (Salin et al., 1996), and lateral amygdala (Huang and Kandel, 1998). In all of these pathways, LTP is expressed presynaptically. Our results reinforce the notion that PKA-dependent LTP is presynaptic. It is reasonable to assume that a $\mathrm{Ca}^{2+}$-sensitive adenylyl cyclase should be present in the neurons that express this form of LTP. Indeed, mRNA for adenylyl cyclases are expressed at high levels in cerebellar granule cells, hippocampal dentate granule cells, and neocortical cells from which corticothalamic fibers originate (Glatt and Snyder, 1993). Presynaptic PKA-dependent LTD has also been recently described in mossy fibers (Kobayashi et al., 1996; Tzounopoulos et al., 1998). Further work will need to determine whether corticothalamic LTD is also mediated by similar mechanisms. Lack of postsynaptic involvement in LTP was also evidenced by the fact 
that LTP was triggered in neurons that did not produce either low-threshold calcium spikes or orthodromic and antidromic action potentials with the synaptic stimulation used. At the network level, activation of $n R t$ neurons and the release of GABA in the ventrobasal thalamus seem to be unnecessary for LTP induction. This is suggested by the observation that glutamate receptor antagonists, which block corticothalamic fiber collaterals in $\mathrm{nRt}$ and thus GABA release, do not impede LTP induction.

The thalamus is the main relay station of information to the neocortex, which then feeds back to the thalamus through corticothalamic fibers. The existence of bi-directional long-term synaptic plasticity in this massive corticothalamic feedback provides an activity-dependent mechanism to enhance or depress the efficacy of communication between the neocortex and thalamus. Because the ventrobasal thalamus feeds back to the neocortex, LTP and LTD would provide the means to change the gain of information flow in the recurrent corticothalamic loop, thus modifying cortico-cortical communication via the thalamus.

When do corticothalamic neurons discharge at $10 \mathrm{~Hz}$ (which produces LTP) or at $1 \mathrm{~Hz}$ (which reverses LTP)? In whole animals, spindle oscillations occur at $10 \mathrm{~Hz}$ during drowsiness and the early stages of sleep. However, spindles are generated within the thalamus (for review, see Steriade et al., 1993, 1997). Instead, during $\alpha$-rhythms, $10 \mathrm{~Hz}$ activity is generated within the neocortex (Lopes da Silva et al., 1980). These cortical rhythms at $10 \mathrm{~Hz}$ occur during awake immobility just as an animal prepares for attentive processing but are abolished immediately after the animal begins active exploration (Rougeul-Buser and Buser, 1997). It is tempting to suggest that one of the functional roles of these cortical preparatory rhythms is to enhance the effectiveness of corticothalamic communication by potentiating corticothalamic synapses. Interestingly, $1 \mathrm{~Hz}$ slow oscillations, which would reverse LTP, are generated in the neocortex during slow-wave sleep (Steriade et al., 1993). One of the functions for cortical slow oscillations during sleep may be to reset (reduce) the efficacy of corticothalamic synapses.

\section{REFERENCES}

Agmon A, Connors BW (1991) Thalamocortical responses of mouse somatosensory (barrel) cortex in vitro. Neuroscience 41:365-379.

Bear MF, Malenka RC (1994) Synaptic plasticity: LTP and LTD. Curr Opin Neurobiol 4:389-399.

Bliss TV, Collingridge GL (1993) A synaptic model of memory: longterm potentiation in the hippocampus. Nature 361:31-39.

Bourassa J, Pinault D, Deschenes M (1995) Corticothalamic projections from the cortical barrel field to the somatosensory thalamus in rats: a single-fibre study using biocytin as an anterograde tracer. Eur J Neurosci 7:19-30.

Castro-Alamancos MA, Donoghue JP, Connors BW (1995) Different forms of synaptic plasticity in somatosensory and motor areas of the neocortex. J Neurosci 15:5324-5333.

Deschenes M, Hu B (1990) Electrophysiology and pharmacology of the corticothalamic input to lateral thalamic nuclei: an intracellular study in the cat. Eur J Neurosci 2:140-152.

Diamond ME (1995) Somatosensory thalamus of the rat. In: Cerebral cortex, Vol 11(Jones EG, Diamond IT, eds), pp189-219. New York: Plenum.

Eaton SA, Salt TE (1996) Role of $N$-methyl-D-aspartate and metabotropic glutamate receptors in corticothalamic excitatory postsynaptic potentials in vivo. Neuroscience $73: 1-5$.

Ergenzinger ER, Glasier MM, Hahm JO, Pons TP (1998) Cortically induced thalamic plasticity in the primate somatosensory system. Nat Neurosci 1:226-229.

Frigyesi TL (1972) Intracellular recordings from neurons in dorsolateral thalamic reticular nucleus during capsular, basal ganglia and midline thalamic stimulation. Brain Res 48:157-172.
Glatt CE, Snyder SH (1993) Cloning and expression of an adenylyl cyclase localized to the corpus striatum. Nature 361:536-538.

Golshani P, Warren RA, Jones EG (1998) Progression of change in NMDA, non-NMDA, and metabotropic glutamate receptor function at the developing corticothalamic synapse. J Neurophysiol 80:143-154.

Guillery RW (1969) A quantitative study of synaptic interconnections in the dorsal lateral geniculate nucleus of the cat. Z Zellforsch Mikrosk Anat 96:39-48.

Guillery RW (1995) Anatomical evidence concerning the role of the thalamus in corticocortical communication. A brief review. J Anat 187:583-592.

Hoogland PV, Wouterlood FG, Welker E, Van der Loos H (1991) Ultrastructure of giant and small thalamic terminals of cortical origin: a study of the projections from the barrel cortex in mice using Phaseolus vulgaris leuco-agglutinin (PHA-L). Exp Brain Res 87:159-172.

Huang Y-Y, Kandel ER (1998) Postsynaptic induction and PKA dependent expression of LTP in the lateral amygdala. Neuron 21:169-178.

Huang Y-Y, Li X-C, Kandel ER (1994) cAMP contributes to mossy fiber LTP by initiating both a covalently mediated early phase and macromolecular synthesis-dependent late phase. Cell 79:69-79.

Kao C-Q, Coulter DA (1997) Physiology and pharmacology of corticothalamic stimulation-evoked responses in rat somatosensory thalamic neurons in vitro. J Neurophysiol 77:1661-1676.

Kobayashi K, Manabe T, Takahashi T (1996) Presynaptic long-term depression at the hippocampal mossy fiber-CA3 synapse. Science 273:648-650.

Levesque M, Gagnon S, Parent A, Deschenes M (1996) Axonal arborizations of corticostriatal and corticothalamic fibers arising from the second somatosensory area in the rat. Cereb Cortex 6:759-770.

Lindstrom S, Wrobel A (1990) Frequency dependent corticofugal excitation of principal cells in the cat's dorsal lateral geniculate nucleus. Exp Brain Res 79:313-318.

Lopes da Silva FH, Vos JE, Mooibroek J, Van-Rotterdam A (1980) Relative contributions of intracortical and thalamocortical processes in the generation of alpha rhythms, revealed by partial coherence analysis. Electroencephalogr Clin Neurophysiol 50:449-456.

McCormick DA, von Krosigk M (1992) Corticothalamic activation modulates thalamic firing through glutamate "metabotropic" receptors. Proc Natl Acad Sci USA 89:2774-2778.

Nicoll RA, Malenka RC (1995) Contrasting properties of two forms of long-term potentiation in the hippocampus. Nature 377:115-118.

Ojima H (1994) Terminal morphology and distribution of corticothalamic fibers originating from layers 5 and 6 of cat primary auditory cortex. Cereb Cortex 4:646-663.

Robson JA (1983) The morphology of corticofugal axons to the dorsal lateral geniculate nucleus in the cat. J Comp Neurol 216:89-103.

Rougeul-Buser A, Buser P (1997) Rhythms in the alpha band in cats and their behavioural correlates. Int J Psychophysiol 26:191-203.

Salin PA, Scanziani M, Malenka RC, Nicoll RA (1996) Distinct shortterm plasticity at two excitatory synapses in the hippocampus. Proc Natl Acad Sci USA 93:13304-13309.

Scharfman HE, Lu SM, Guido W, Adams PR, Sherman SM (1990) $\mathrm{N}$-methyl-D-aspartate receptors contribute to excitatory postsynaptic potentials of cat lateral geniculate neurons recorded in thalamic slices. Proc Natl Acad Sci USA 7:4548-4552.

Sherman SM, Guillery RW (1996) Functional organization of thalamocortical relays. J Neurophysiol 76:1367-1395.

Steriade M, Timofeev I (1997) Short-term plasticity during intrathalamic augmenting responses in decorticated cats. J Neurosci 17:3778-3795.

Steriade M, Wyzinski P (1972) Cortically elicited activities in thalamic reticularis neurons. Brain Res 42:514-520.

Steriade M, McCormick DA, Sejnowski TJ (1993) Thalamocortical oscillations in the sleeping and aroused brain. Science 262:679-685.

Steriade M, Jones EG, McCormick DA (1997) Thalamus, Vol 1. New York: Elsevier.

Tsumoto T, Creutzfeldt OD, Legendy CR (1978) Functional organization of the corticofugal system from visual cortex to lateral geniculate nucleus in the cat (with an appendix on geniculo-cortical mono-synaptic connections). Exp Brain Res 32:345-364.

Turner JP, Salt TE (1998) Characterization of sensory and corticothalamic excitatory inputs to rat thalamocortical neurons in vitro. J Physiol (Lond) 510:829-843.

Tzounopoulos T, Janz R, Sudhof TC, Nicoll RA, Malenka RC (1998) A role for cAMP in long-term depression at hippocampal mossy fiber synapses. Neuron 21:837-845. 
Vidnyanszky Z, Gorcs TJ, Negyessy L, Borostyankoi Z, Kuhn R, Knopfel $\mathrm{T}$, Hamori J (1996) Immunocytochemical visualization of the mGluR1a metabotropic glutamate receptor at synapses of corticothalamic terminals originating from area 17 of the rat. Eur J Neurosci 8:1061-1071.

Wang JH, Kelly PT (1996) Regulation of synaptic facilitation by postsynaptic $\mathrm{Ca}^{2+} / \mathrm{CaM}$ pathways in hippocampal CA1 neurons. J Neurophysiol 76:276-286.

Weinberger NM (1995) Dynamic regulation of receptive fields and maps in the adult sensory cortex. Annu Rev Neurosci 18:129-158.

Weisskopf MG, Castillo PE, Zalutsky RA, Nicoll RA (1994) Mediation of hippocampal mossy fiber long-term potentiation by cyclic AMP. Science 265:1878-1882.
Wigstrom H, Gustafsson B (1983) Facilitated induction of hippocampal long-lasting potentiation during blockade of inhibition. Nature 301:603-604.

Yuan B, Morrow TJ, Casey KL (1985) Responsiveness of ventrobasal thalamic neurons after suppression of S1 cortex in the anesthetized rat. J Neurosci 5:2971-2978.

Zalutsky RA, Nicoll RA (1990) Comparison of two forms of long-term potentiation in single hippocampal neurons. Science 248:1619-1624.

Zhang ZW, Deschenes M (1997) Intracortical axonal projections of lamina VI cells of the primary somatosensory cortex in the rat: a single-cell labeling study. J Neurosci 17:6365-6379.

Zucker RS (1989) Short-term synaptic plasticity. Annu Rev Neurosci 12:13-31. 